\title{
Detection and Classification of Selected Noise Sources in Long-Term Acoustic Climate Monitoring
}

\author{
M. KŁACZYŃSKI* AND T. WSZOŁEK \\ AGH University of Science and Technology, Faculty of Mechanical Engineering and Robotics \\ Department of Mechanics and Vibroacoustics, al. A. Mickiewicza 30, 30-059 Krakow, Poland
}

\begin{abstract}
Continuous acoustical climate monitoring of the environment raises several problems related to large quantities of the recorded data, which often represents information unrelated to the studied noise source. Manual verification of such data is time-consuming and costly. Therefore, developing effective methods for automatic identification of transport noise sources becomes an important task for the proper determination of noise levels. This paper presents a concept of such method of automatic detection and classification of the noise sources from the air and railway transportation in the acoustic environmental monitoring.
\end{abstract}

PACS: 43.60.Lq, 84.35.+i, 43.55.Ka

\section{Introduction}

Over the last two decades in Poland, the country's development of transportation became one of the main sources of the environmental noise hazards. The road noise has been a dominant factor of the acoustic climate, due to its widespread nature and prolonged effect. Also such other sources of noise associated with air transportation, as flights, take-off and landing of aircrafts, as well as noise generated by the railway industry are also of great significance.

The European Union established a regulatory framework with uniform requirements for the assessment and management of environmental noise in Directive 2002/ $49 /$ EC. Poland implemented provisions of the said Directive into the Environmental Protection Law Act of 27 April 2001 (as amended). The purpose of this legislation is protection of health, quality of life and well-being of the population. While exploiting the environment by emission of significant quantities of acoustic energy, management of a road, a railway, a tram-line, an airport, or a harbor is required to continuously monitor such emissions. Such data gathering and studies are designed to collect information about the prevailing acoustic climate and to produce conclusions, reports and maps of the areas most threatened with the limits being exceeded. Carrying out continuous monitoring of a particular area, involves issues of large quantities of the recorded data, often representing information unrelated to the study source. Manual verification of such data is time-consuming and costly. Therefore, developing ef-

* corresponding author; e-mail: maciej.klaczynski@agh.edu.pl fective methods for automatic identification of transport noise sources becomes an important task for the proper determination and expanding knowledge regarding the causes of changes in noise levels in the environment and present trends. This paper presents such a method as applied to the automatic detection of air and railway noise during long-term acoustic monitoring of the environment.

\section{Detection of acoustic events}

Methodology for measuring road noise is relatively simple and the use of automatic noise monitoring station does not involve much difficulty, and the traffic occurs continuously. The detection of traffic event can be supported by special microwave sensors such as SmartSensor HD [1] or Remote Traffic Microwave Sensor [2]. The aircraft noise measurements are more complicated and their range includes large areas. In such area, usually defined as zone of the limited use, there are often other noise sources present, such as roads or railway lines then treated as a background sound. In such case, it is important to distinguish automatically a source of noise from the background and to quantify the impact various sources on the overall noise level in a given place.

Currently there are available aircraft noise monitoring stations using four-microphone array with the task to determine the source position (angle of elevation and azimuth) and a fifth microphone to sound level measurements [3] or using the information from airport radar system [4]. Number of needed microphones in array or payment for using of radar system greatly increases the cost of such devices. Thus, there arises the idea of the use of automated pattern recognition methods for identification of acoustic noise sources and of the type of airplane 
operations. The current technology is developed enough to create an efficient algorithm and its hardware implementation seems to be possible as well.

In principle, all applied methods of automatic classification, including pattern recognition depend on the following problem being solved: the solutions' lack of versatility results not from imperfection of the recognition methods, but rather from excessive complexity of source signals. For this reason, special transformation of the signals analyzed is applied in order to obtain an appropriate simple feature space. Currently, although a number of specific solutions exist, two principal methods to creating the space of features can be distinguished. The first one consists of the search of the suitable transformation of the signal and using its results (parameters) as the signal characteristics. The other approach consists of the search of a model describing the way of the observed signals' emission, e.g. identification of filters generating the signal. The problem discussed can be expanded to cover also the noise immission or specifically perception of the observed signal or, more precisely, perception of the observed signal.

There are many methods of a pattern recognition, which are dealing with an abstract pattern notion, understood as a set of certain features and relations (Fig. 1). Several algorithms and instruments recognizing certain determined classes of objects were developed [5-8]. However, in practical applications, it is not clear which features and relations should be counted as the basic ones, and how to single them out from the recognized patterns. It was assumed that in order to obtain features needed at the recognition, the preliminary signal processing should occur in an identical fashion just as in visual and audio systems of humans.

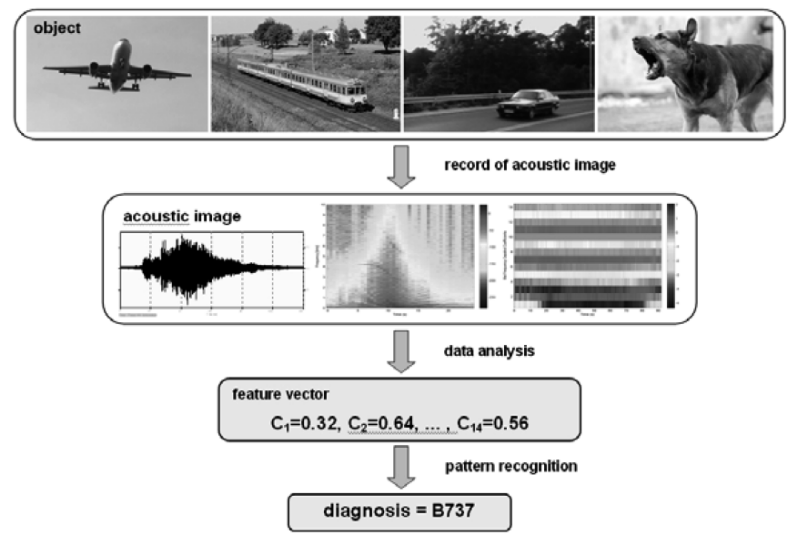

Fig. 1. Model of acoustic pattern recognition.

Most researchers agree that the features necessary for pattern recognition of sound should be sought in a time-frequency domain of an acoustic signal. The point for the decision to apply the parameters of the acoustic signal in the mel frequency scale was the observation that virtually everyone who had any contact with vehicles and transport facilities is able to detect their presence and dis- tinguish their type (airplane, train, tram, car, truck, motorcycle) on basis of perceived auditory experience during their movement. The cepstral analysis in mel scale is based on the characteristics of the human hearing, thus the authors decided to use it in their research. Mel frequency cepstral coefficients (MFCC) analysis is a widely used algorithm in nonstationary signals study (including the recognition and speech intelligibility in communication systems). The human ear distinguishes sounds using non-linear frequency scale of spectrum. This scale is linear only to $1 \mathrm{kHz}$, while the non-linearity for the higher frequencies can be described using a logarithmic scale [9]. Practical importance of cepstrum is that it often enables more transparent interpretation of the information contained in spectrum. This applies in particular to assess of the relationship of spectral frequency components contained in the signal. A specific feature of cepstrum is its ability to separate effects related to operation of the sound source from the effects related with the transmission (propagation path) [10]. This fact is very important, because depending on location of monitoring stations (terrain, season of year, weather conditions) are changing acoustic wave propagation between the source and receiver.

\section{Research}

The recordings from the mobile station of long-term acoustics monitoring shown in Fig. 2 have been used while classifying the traffic noise - air operations, movement of trains. The location of station include: village Rząska near Kraków (the measuring point in the close location of a railway line and route of departures and arrivals to "Kraków Airport") and village Morawica near Kraków (the measuring point in the vicinity of "Kraków Airport" and motorway A4). In this research the following procedures of the digital signal processing were applied in calculations:

- recording signals with sampling frequency $f_{\mathrm{s}}=$ $48 \mathrm{kHz}$;

- decimation of signal;

- Hamming's time window of a length $N=12000$ samples;

- 12000 points fast Fourier transform (FFT) calculated every $500 \mathrm{~ms}$ of signal;

- rescaling the frequency scale into the mel frequency scale, according to the following:

$$
\operatorname{mel}(f)=2595 \log \left(1+\frac{f}{700}\right) ;
$$

- mel filtration - it means the spectrum conversion into the form of midband pass filters (summing the weighted individual spectral lines, where the coefficients of corresponding triangle filters are used as the weights). The number of filters in the set: $N=14$; 
- determination of cepstral coefficients in the mel frequency scale (MFCC) as discrete conversion of logarithm cosinuses of the parameters of filter data, according to the formula:

$$
C_{n}=\sqrt{\frac{2}{N}} \sum_{i=1}^{N} \log \left(s_{i}\right) \cos \left(\frac{\pi n}{N}\left(i-\frac{1}{2}\right)\right),
$$

where $C_{n}-n$-th cepstral coefficient, $n=1, \ldots, 14$, $S_{i}-i$-th coefficient obtained from signal conversion by the set of filters, $N$ - number of filters in the set, $N=14$.
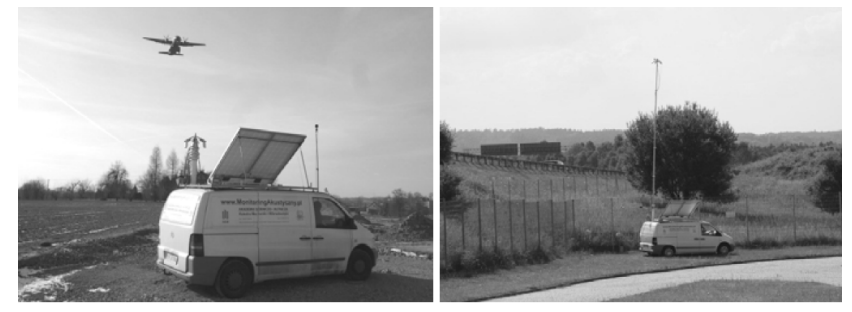

Fig. 2. The mobile station of long-term acoustic monitoring.

Analysis of the acoustic signal emitted by the aircraft was limited to selected types: B737, A320, ATR72, ATR42, AN24, C-295. However, the analysis of railway noise was limited to the types of locomotives: EU06, EU07, ET21, ST43, ST44, and train EN57. In some previous papers [11-13] many variants of the parameters feature vectors has been presented as an analysis and discussion of the correctness in selection of the feature space. Proposed new feature vector is based on mel frequency cepstral coefficients and is defined below Eq. (3.3):

$$
\left\langle C_{1}, C_{2}, \ldots, C_{14}\right\rangle=X .
$$

For example, in Fig. 3 a comparison of mel frequency cepstral coefficients for noise events representing three classes, the source of aircraft noise - B737 jet flight, railway noise - a ride of train with a locomotive EN57, background sound - a drive of a car, a dog barking is shown.

The research concerning recognition of aircraft noise has been carried out by two groups of methods: pattern recognition - the minimum distance method (with Euclidean metric) and neural network techniques. In the group of pattern recognition methods the nearest neighbor $(\mathrm{NN}), k$ nearest neighbors $(\mathrm{kNN})$ and nearest mode (NM) have been applied [5]. In neural network techniques, the choice of network architecture (number of layers and the number of neurons in each layer), as well as the activation function of neurons and learning networks method were made in an experimental way. The best performance was characterized by a nonlinear three-layer network, having the characteristics of neurons, sigmoidal and hyperbolic tangent as activation functions. The network was trained using error back propagation with a decrease in the gradient. The experiment used $70 \%$ of

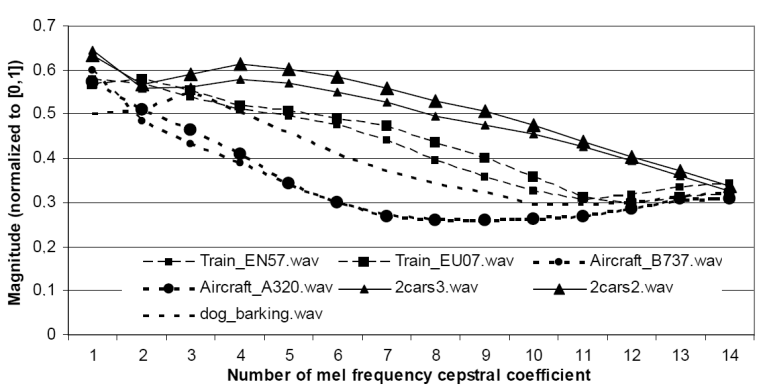

Fig. 3. Comparison of mel frequency cepstral coefficients for noise events representing three classes (source of aircraft noise - flight of aircraft, source of railway noise - a train ride, background - the car drive, dog barking).

the recorded material to the learning network, and $30 \%$ of the material to verify the method. The results identify the noise sources due to its type, shown in Table, in which aircraft noise source is an aircraft, railway noise source is a train. Acoustic pattern recognition method based on artificial neural network technique has the highest value in comparison with used in the study, the minimum distance methods ( $\mathrm{NN}$ and $\mathrm{kNN}, \mathrm{NM}$ ).

TABLE

Recognition results of aircrafts and railway noise events.

\begin{tabular}{l|c|c}
\hline \hline \multirow{2}{*}{ Methods of recognition } & \multicolumn{2}{|c}{ Recognition correctness [\%] } \\
\cline { 2 - 3 } & Aircraft & Train \\
\hline NN (nearest neighbor) & 80 & 82 \\
kNN ( $k$ nearest neighbor) & 88 & 91 \\
NM (nearest mode) & 87 & 90 \\
neural network & 94 & 96
\end{tabular}

\section{Conclusions}

This paper presents a method of automatic identification of aircraft and railway noise sources in the environment acoustic monitoring systems. The mentioned method relies on automatic "meaning" of sounds recorded microphone technique. The proposed algorithm is based on artificial intelligence techniques using mel frequency cepstral coefficients of signals. The developed algorithm can be incorporated into existing monitoring systems of acoustic climate, and also used in the analysis of recordings from existing systems and as other solutions - such as acoustic radar, while recognizing the nature and type of aircraft or flight operation, or a train movement. The condition is to collect a representative sample group of patterns and effectively trained neural network.

\section{Acknowledgments}

The paper has been written and the respective research undertaken within the project R03 0030 06/2009 (National Centre for Research and Development). 


\section{References}

[1] SmartSensor HD Technical Specification, Wavetronix, USA, 2011, http://ww . wavetronix.com/en/ products/smartsensor/hd/specs .

[2] Remote Traffic Microwave Sensor, Image Sensing Systems Canada Ltd, 2011, http://www.imagesensingca.com/en/RTMS/ Specifications/tabid/68/Default.aspx .

[3] Environmental Sound Monitoring (For Aircraft Noise Measurement), Rion, Japan, 2011, http://www .rion.co.jp/dbcon/pdf/NA-37-E.pdf .

[4] Airport Environment Management, Bruel \& Kjaer, Denmark, 2011, http://www.bksv.com/ doc/bn0636.pdf, http://www.bksv.com/doc/ bp1883.pdf .

[5] R. Tadeusiewicz, M. Flasiński, Pattern Recognition, PWN, Warszawa 1991, p. 32 (in Polish).
[6] R. Tadeusiewicz, Neural Networks, AOW, Warszawa 1993, p. 13 (in Polish).

[7] M. James, Classification Algorithms, Wiley, New York 1985, p. 1.

[8] R.O. Duda, P.E. Hart, D.G. Stork, Pattern Classifications, 2nd ed., Wiley, New York 2001, p. 9.

[9] L. Rabiner, B.H. Juang, Fundamentals of Speech Recognition, Prentice Hall, New Jersey 1993, p. 183.

[10] T. Zieliński, Digital Signal Processing, WKE, Warszawa 2005, p. 555 (in Polish).

[11] W. Wszołek, M. Kłaczyński, T. Wszołek, in: InterNoise 2010, Lizbon (Portugal), 2010, p. 330, CD.

[12] W. Wszołek, R. Tadeusiewicz, A. Chyla, in: InterNoise 2001, Hague (Netherlands), 2001, p. 2217, CD.

[13] W. Wszołek, R. Tadeusiewicz, in: InterNoise 2005, Rio de Janeiro (Brazil), 2005 p. 69, 1797, CD. 